\title{
Anpassung an die Medienlogik? Eine Untersuchung politischer Kommu- nikationsstrategien von AkteurInnen aus Deutschland und Österreich
}

\author{
Andreas Riedl ${ }^{1, *}$, Peter Maurer ${ }^{2}$, Birgit Mühl ${ }^{3}$ \\ 1 Institut für vergleichende Medien- und Kommunikationsforschung, Österreichische Akademie der Wissenschaften und Alpen-Adria- \\ Universität Klagenfurt, Austria \\ 2 Institut für Soziologie und Politikwissenschaft, Technisch-Naturwissenschaftliche Universität Trondheim, Norway \\ 3 Institut für Publizistik- und Kommunikationswissenschaft, Universität Wien, Austria \\ * Andreas.Riedl@oeaw.ac.at
}

\section{Zusammenfassung}

Obwohl die steigende Abhängigkeit politischer Eliten von öffentlicher Kommunikation sowie ihre Orientierung an medialen Formaten überzeugend postuliert werden, gibt es wenige empirische Erkenntnisse dazu, wie sich dies in ihren Vorstellungen niederschlägt. Deshalb fragt dieser Beitrag, inwieweit sich die Wahrnehmungen von PolitikerInnen und KommunikationsberaterInnen in Deutschland und Österreich bezüglich der Effektivität medienlogikorientierter Kommunikationsstrategien inter-individuell und im Ländervergleich unterscheiden. Er stützt sich auf eine Sekundäranalyse von 259 offenen Antworten von 318 Befragten aus einer internationalen Befragung zur politischen Kommunikationskultur (2008/2009). In diesen wurden 592 Einzelstrategien genannt, die dann induktiv zu Kategorien verdichtet und quantitativ ausgewertet wurden. Eine bivariate Auswertung belegt dabei relative Homogenität zwischen Ländern und Regierungs- bzw. Oppositionsparteien bei leicht stärkerer Tendenz zur Dramatisierung in Österreich. Eine multiple logistische Regressionsanalyse zeigt jedoch, dass die Parteizugehörigkeit sowie geringes Vertrauen in parlamentarische Kommunikation die Wahl medienlogischer Strategien begünstigen.

\section{Schlüsselwörter}

Mediatisierung, politische AkteurInnen, Medienlogik, Kommunikationsstrategie, Vergleich, Methodenmix

\section{Adoption of Media Logic? An Investigation of Political Communication Strategies of Actors in Germany and Austria}

\begin{abstract}
Although it is theoretically claimed that political elites get increasingly dependent on public communication and stronger orientated towards media logic formats, empirical knowledge to underpin this claim is scarce. Therefore, this paper asks how far perceptions of the effectiveness of different political communication strategies reflecting fundamentals of media logic differ between German and Austrian politicians and spokespersons. The study is based on 259 answers to open questions by 3 I 8 respondents surveyed in an international study about political communication cultures (2008/2009). The answers were dissected into 592 single communicative strategies, which were then inductively condensed and counted. A bivariate quantitative analysis indicates a relative homogeneity between countries and actors belonging to either governing or opposition parties. Only a slightly stronger tendency towards dramatizing a story was found in Austria. Nonetheless, a multiple logistic regression analysis shows that the specific party a respondent belongs to and his/her low trust in the effectiveness of parliamentary speeches increase the probability to value strategies geared toward media logic.
\end{abstract}

\section{Keywords}

mediatization, political actors, media logic, communication strategies, cross country comparison, mixed methods

The authors have declared that no competing interests exist.

March 31, 2018 I innsbruck university press, Innsbruck 


\section{Einleitung}

Kommunikation nimmt im Rahmen der Artikulation und Durchsetzung politischer Interessen einen hohen und wachsenden Stellenwert ein. Gerade durch die Abschwächung von festen Parteibindungen, die Personalisierung der Wahlkampfführung (Brettschneider 2002a; Plasser/Ulram 2006), die steigende Unzufriedenheit mit politischen Entscheidungen seitens verschiedener Anspruchsgruppen und das (trotz Schwankungen) immer wieder konstatierte Desinteresse an Politik in gesättigten Demokratien (Heil 20I6) ist der Erwerb politischer Legitimität „kommunikationsabhängiger" (Sarcinelli 20II, IO3) geworden. Damit ist gemeint, dass politische AkteurInnen zur Mobilisierung von Unterstützung in besonderer Weise auf die Beeinflussung des öffentlichen Diskurses angewiesen sind. Wenn Wahlausgänge zunehmend von der Themenagenda der Medien abhängig sind (Brettschneider 2002b), ist es für politische AkteurInnen geradezu überlebenswichtig, ,ihre themen in die öffentliche Diskussion einzubringen und Aufmerksamkeit dafür zu schaffen (Marcinkowski/Steiner 2009). Dazu sind sie auf effektive Strategien politischer Kommunikation angewiesen. Vor diesem Hintergrund ist es erstaunlich, dass politische Kommunikationsstrategien, die darüber Auskunft geben, wie PolitikerInnen versuchen, politische Debatten mitzugestalten, bislang kaum untersucht wurden (Seiffert/Fähnrich 2015).

Solche Strategien können vielfältig sein, doch kann man annehmen, dass sie sich an den Strukturen und AkteurInnen, welche Kanäle für öffentliche Kommunikation bereitstellen, orientieren. Auch im Zeitalter des Internets sind immer noch klassische Medienorganisationen wie Fernsehsender und reichweitenstarke Printmedien sowohl die Hauptproduzenten von Nachrichten, als auch primäre Informationsquellen der WählerInnen (Shehata/Strömbäck 20I4). Somit sind sie auch aus Sicht politischer AkteurInnen wichtige Bezugspunkte und Restriktionen. Weil sie darauf angewiesen sind, dass ihre Botschaften die Schleusen der medialen Gatekeeper passieren, wird in der Mediatisierungsforschung ${ }^{\mathrm{I}}$ von einer Abhängigkeit politischer AkteurInnen von den Medien ausgegangen (z. B. Mazzoleni/Schulz, 1999; Strömbäck 2008). Dieser Sichtweise zufolge entscheiden Medien heutzutage weitgehend autonom, entsprechend einer eigenen (Medien-)Logik, ob und wie sie über politische Inhalte berichten. Theoretisch wurde vielfach argumentiert, dass im Zuge der fortschreitenden Mediatisierung politische AkteurInnen, eben weil sie auf die Vermittlungsleitung autonomer Medien angewiesen sind, diese aber nicht (mehr) kontrollieren können, dazu übergehen, ihre Kommunikation an deren Logik anzupassen

I Mediatisierung und Medialisierung werden hier Reinemanns (20IO, S. 280) Argument, dass die beiden Begriffe meist synonym verwendet werden, folgend konzeptionell gleichbehandelt.
(Strömbäck 2008). Empirische Anhaltspunkte dafür gibt es jedoch erst wenige (Strömbäck/Esser 20I4).

Wenn überhaupt, so sind sowohl politische Kommunikationsstrategien als auch die Auswirkungen der Mediatisierung bisher vorwiegend für politische Organisationen (Donges 2008; Seiffert/Fähnrich 2015) untersucht worden. Demgegenüber stellt dieser Beitrag individuelle PolitikerInnen sowie ihre SprecherInnen und KommunikationsberaterInnen in den Vordergrund. Die individuelle Ebene ist bei der Erforschung strategischer politischer Kommunikation unter dem Druck der Mediatisierung bisher nur wenig beachtet worden. Dies ist aber nicht zuletzt deshalb notwendig, weil Kommunikationsstrategien sich im Zuge der Personalisierung, Modernisierung und Mediatisierung der Politik stärker individualisieren. Man kann deshalb vermuten, dass sich das Problem, effektiv zu kommunizieren, auf die einzelnen PolitikerInnen verlagert und sich für sie verschärft darstellt. Politik wird in modernen Demokratien, nicht zuletzt aufgrund eines stärker personalisierten Wählerverhaltens, immer stärker durch Einzelpersonen vermittelt. Aus diesem Grund kommt dem Stil von Einzelpersonen auch eine große Bedeutung für den Erfolg politischer Kommunikation zu. Deshalb rückt die Frage, welche Strategien einzelne politische AkteurInnen wählen, besonders in den Blickpunkt. Insbesondere stellt sich die Frage, inwieweit sie ihre strategische politische Kommunikation an die Medienlogik anpassen, um politisch durchsetzungsfähig zu sein.

In diesem Beitrag soll diese Anpassung auf individueller Ebene auf Basis einer Befragung von politischen AkteurInnen verschiedener Parteien und Länder - den modernen, parlamentarischen Demokratien Deutschland und Österreich - die in unterschiedlichen Rollen und Funktionen agieren, untersucht werden. Der Fokus der Untersuchung liegt auf ihrem individuellen, strategischen Kommunikationsverhalten zur Beeinflussung des öffentlichen Diskurses. Wir konzentrieren uns dabei auf die Frage, ob und inwieweit sich ihre Einstellungen zur Effektivität an der Medienlogik orientierter Kommunikationsstrategien inter-individuell unterscheiden.

\section{Medienlogik und politische Kommunikations- strategien}

Der Prozess der Mediatisierung der Politik ist eng mit der Vorstellung eines verstärkten Einfließens der sogenannten Medienlogik in politisches Denken und Handeln verknüpft. So argumentiert Strömbäck (2008), dass in einer ersten Phase der Mediatisierung zunächst die Frage im Zentrum steht, inwiefern Medien die dominante politische Informationsquelle darstellen. Während in der zweiten Phase Medien einen Prozess der Professionalisierung durchlaufen und Politik und Medien in eine 
gegenseitige Abhängigkeit gelangen, übernehmen $\mathrm{Me}$ dien in der dritten Phase die Oberhand - politische AkteurInnen sind zusehend gezwungen, sich deren Logik anzupassen. In der vierten Phase gehen sie schließlich dazu über, diese Logik so weit in ihr eigenes Denken und Handeln zu übernehmen, dass sie die politische Handlungslogik substituiert. Strömbäck spricht hier vom Übergang von einer adaption (strategischen Anpassung) der Medienlogik hin zur adoption (kompletten Aneignung ihrer Gesetze) (2008, 240). Das Raumgreifen der Medienlogik in politischen Prozessen aufgrund dessen, dass sich politische AkteurInnen die Funktionslogik der Medien aneignen, wird demnach als wichtigster Treiber, als eigentlicher Kern der Mediatisierung gesehen (Altheide 20I4).

Auf der Mikro-Ebene kommt Mediatisierung im Zuge einer sogenannten Selbstmediatisierung von politischen AkteurInnen zum Tragen. In diesem „process of self-initiated stagemanagement and media-friendly packaging" (Esser 2013, I62) ordnen sie sich intentional medialen Imperativen unter. Im Zuge dieser „mentalen Medialisierung" (Marcinkowski 2015) haben Medien damit Effekte auf die (potentiellen) Objekte ihrer Berichterstattung und nicht nur auf Rezipierende. Dabei ist insofern von einem „Primat der Antizipation über den Inhalt" (Marcinkowski 2015, 89) auszugehen, als dass allein der Umstand des möglichen Beobachtetwerdens die Einstellung der politischen AkteurInnen gegenüber den Medien determiniert; eine Analyse des Stellenwerts von Medienlogik für PolitikerInnen setzt also am besten auf der Ebene der Perzeptionen an.

Fortgeschrittene Mediatisierung politischer AkteurInnen lässt sich zusammenfassend also als Anpassung von politischem Handeln und den diesem vorgelagerten Wahrnehmungen und Urteilen an die Medienlogik beschreiben. Dies bewirkt konkret, „dass sich die Kriterien und Rationalitäten verändern, nach denen AkteurInnen handeln und entscheiden" (Reinemann 20IO, 280). Der Begriff "Medienlogik" selbst wurde dabei von Altheide und Snow (1979) geprägt. Ihrer Definition zufolge sind damit die verschiedenen Formate der Massenmedien gemeint, inklusive der ungeschriebenen Regeln, nach denen JournalistInnen (politisches) Rohmaterial auswählen und zu Nachrichtenprodukten umformen. Die Organisation der enthaltenen Informationen und der jeweilige Präsentationstil gehören zu den Grundelementen dieser Regeln, die mit einer Art „Grammatik“ (Altheide/Snow I979, IO) gleichgesetzt werden.

Allerdings greift ein Verständnis der Medienlogik, das sich bloß auf die Darstellungsregeln der Medien selbst bezieht, zu kurz, denn Medienlogik besitzt einen reflexiven Charakter. Sie manifestiert sich folglich nicht nur in der medialen Darstellung von Politik, sondern auch im Kommunikationsverhalten der politischen AkteurInnen, wenn sie die medialen Selektions- und Dar- stellungsregeln anerkennen und in ihre Kommunikationsstrategien inkorporieren (Altheide 2014). Esser (2013, I67) begreift Medienlogik in einem weiteren Verständnis demnach als "way of covering and interpreting social, cultural, and political phenomena" und trägt damit dem Umstand Rechnung, dass die Formate bestimmter Mediengattungen die Konstruktion politischer Botschaften schon bei ihren Absendern beeinflussen.

Davon ausgehend ergibt sich die zentrale Frage, woran sich erkennen lässt, ob und wie stark sich politische AkteurInnen in ihrem Kommunikationsverhalten einer Medienlogik anpassen. Dies kann am besten daran abgelesen werden, wie sie glauben, ihre Botschaften gestalten zu müssen, um eine gewünschte Wirkung zu erzielen. Je stärker politische AkteurInnen davon überzeugt sind, ihre Botschaften entsprechend den Regeln der Medienlogik gestalten zu müssen, desto stärker passen sie sich folglich dieser Logik an.

Wir argumentieren folglich, dass stark medienlogikorientierte Strategien daran zu erkennen sind, dass die AkteurInnen ihre Botschaften in erster Linie auf ihre massenmediale Verwertbarkeit und Wirkung hin konzipieren. Schwach medienlogikorientierte Botschaften können dagegen auch in einer nicht-medialen Öffentlichkeit, wie etwa in Versammlungsöffentlichkeiten oder persönlichen Gesprächen, eine Funktion haben und dort eine Wirkung erzielen. Dazu zählen besonders solche, die darauf ausgerichtet sind, durch die Verwendung von rationalen, komplexer aufgebauten Argumenten und sprachlichen Mitteln Aufmerksamkeit und Verständnis für die politische Bedeutung von Themen zu erzeugen. Schon Platon sprach von Persuasion als dem "Gewinnen des menschlichen Geistes durch Worte" (Kuhn 2009, II2) und überlegte, wie dies mit rhetorischen Stilmitteln möglich sei. Dementsprechend orientieren sich schwach medienlogikorientierte politische Kommunikationsinputs nach unserem Verständnis an Gesetzen der politischen Rhetorik und zielen auf politische Wirkungen durch Persuasion mit rationalen Argumenten, die in der Regel in Text- oder Sprachform vermittelt werden. Eine weitere Strategie, die nicht der Medienlogik entspricht, stellt der Versuch dar, die Aufmerksamkeit nicht auf die medialen Gatekeeper, sondern auf gesellschaftliche Verbündete (wie bestimmte Verbände und BürgerInnen) zu richten, um Themen auf die politische Agenda zu bringen.

Mit dem Einsatz nicht-medienlogikorientierter Botschaften wird weniger die Überwindung der medialen Aufmerksamkeitsschwelle als vielmehr die dauerhafte Beeinflussung von politischen Einstellungen angestrebt. Dies ist im Unterschied zum Schaffen von Interesse im Mediensystem durch Antizipation der dort herrschenden Produktionsroutinen ein originär politisches Ziel. Bei nicht-medienlogikorientierten Botschaften hat folglich die formatkompatible Verpackung den inhaltlichen Gehalt nicht in den Hintergrund gedrängt. 
Demgegenüber versuchen stark medienlogikorientierte Botschaften, ihre Wirkung über Theatralität, Inszenierungen und Bildlichkeit zu erzielen. Sie zeichnen sich dadurch aus, dass sich ihre KommunikatorInnen vor allem an Darstellungsweisen und -formaten der audiovisuellen Medien, insbesondere des Fernsehens, orientieren (Altheide 20I4; Meyer 200I; Brettschneider 2002a), um so die Zugangsbarrieren dieser Medien zu durchbrechen. Konkret versuchen sie, als wahrscheinlich angenommene Reaktionen von JournalistInnen auf ihre Botschaften bei deren Gestaltung zu antizipieren, auch wenn dies die inhaltlichen Aussagen nicht schärft, sondern sogar verunklart oder in ihrer Komplexität reduziert (Altheide 2004; Kepplinger 2009). Mit stark medienlogikorientierten Kommunikationsinputs verbinden sich somit Wirkungsabsichten, die primär auf journalistische Gatekeeper zielen, nur mittels Medientechnologie erzielt werden können und ganz auf die Passfähigkeit zu massenmedialen Formaten hin konzipiert sind.

Diese Merkmale stark medienlogikorientierter Botschaften stimmen mit Kennzeichen der von Meyer (200I) beschriebenen Strategien mediatisierter Politik überein. Dabei unterscheidet er drei zentrale Strategien: Eventpolitik, Imageprojektionen und Scheinhandlungen. Symbolische Politik sieht Meyer (200I, II6) als eine Art der strategischen Kommunikation, die „keine Argumente vorträgt und keine [...] Beziehung zwischen ihrem ästhetischen Schein und der Wirklichkeit [...] herstellt". Ihre Intention ist meist in der emotionalen Beeinflussung des Publikums, oft durch Rückgriff auf Bilder (auch Comics oder Verfremdungen), zu sehen. Die Aktivitäten adressieren dementsprechend primär audiovisuelle Medien und kopieren Stilmittel des populären Journalismus. Trotzdem enthalten auch solche Kommunikationsaktivitäten eine politische Logik, die allerdings gegenüber der medialen zurücktritt. Anders gesagt, nehmen die KommunikatorInnen Differenzierungsverluste in Kauf, um die Kompatibilität ihrer Kommunikation mit der Medienlogik und die emotionale Wirkung zu erhöhen. Dementsprechend besteht deren politische Funktion nicht unbedingt im Einführen neuer Argumente, sondern in der Generierung von Aufmerksamkeit und dem Auslösen eher diffuser Gefühle von Vertrauen, Alarmiertheit oder Sympathie.

\section{Stand der empirischen Forschung}

Neben der theoretischen Auseinandersetzung mit der Anpassung des Kommunikationsverhaltens politischer AkteurInnen an die Medienlogik mangelt es an Untersuchungen, die dies auch empirisch nachzeichnen könnten. Solche Befunde zur Verwendung von Strategien der politischen Öffentlichkeitsarbeit gibt es bisher allen- falls für die Gruppe der PR-Treibenden (Tenscher 2003; Pfetsch 2003; Plasser/Hüffel/Lengauer 2004) sowie für LokalpolitikerInnen (Koch/Baden/Klötzer/Müller 2013). Dabei fand Pfetsch (2003) in einer Befragung von PressesprecherInnen Mitte der I990er Jahre für Deutschland heraus, dass die Pflege persönlicher Kontakte zu JournalistInnen, die Vergabe von Zugangsprivilegien an diese, klassische Pressearbeit und die Schaffung mediengerechter Inszenierungen besonders gern gewählte Strategien zur Erzeugung öffentlicher Aufmerksamkeit sind. Plasser, Hüffel und Lengauer (2004) stellten in einer Befragung von führenden Personen aus dem Bereich der politischen PR im Jahr 2003 für Österreich ganz ähnliche Strategien fest. Individuelle Kontaktpflege mit JournalistInnen stellte sich als die wichtigste Strategie heraus. Dazu gehören Hintergrundgespräche und Zusammentreffen in informellen Settings. Die Autoren kommen zu dem Schluss, dass österreichische PressesprecherInnen ,auf ein dichtes Beziehungsmanagement und ein persönliches Nahverhältnis zu führenden Journalisten setzen" (2004, 34I, Herv. i. O.).

Bei einem Trend-Vergleich der Kommunikationsstrategien beim Agenda-Building politischer SprecherInnen in Deutschland zeigte sich, dass im Zeitraum von Mitte der 1990er bis Ende der 20ooer Jahre medienlogikorientierte Strategien gegenüber klassischer Pressearbeit und politisch-orientierten Strategien wichtiger geworden sind (Maurer \& Mayerhöffer, 2009). Eine jüngere Untersuchung zur Professionalität der Kommunikation bayerischer LokalpolitikerInnen vor dem Hintergrund der Mediatisierung ergab, dass eine Mehrheit der Befragten spezifische Kommunikationstrainings absolviert hat oder in Fortbildungen Kompetenzen im Umgang mit Medien vermittelt bekam. Die Befragung ergab außerdem, dass diese Kommunikations- und Medienkompetenz als sehr relevant für ihre Arbeit einschätzen (Koch/Baden/Klötzer/Müller 20I3). Obwohl außer nach dem Einsatz neuer Medien nicht nach konkreten Kommunikationsstrategien gefragt wurde, sehen die Autoren die Ergebnisse als Bestätigung des Zusammenhangs zwischen „Medialisierungsdruck und sich professionalisierendem Kommunikationshandeln im kommunalpolitischen Kontext" (ebd. 290).

In einer standardisierten Befragung verschiedener politischer AkteurInnen zur Mediatisierung im Politikfeld Energiepolitik zeigten deutsche Abgeordnete bei Strategien des Media-Agenda-Building eine starke Orientierung an der Medienlogik (Fawzi 20I4). Zusammenfassend lässt sich aus dieser Untersuchung schließen, dass sich Parteien beim Bearbeiten der Medienagenda besonders an den auch als Nachrichtenfaktoren bedeutenden Aspekten Dramatisierung und Personalisierung orientieren und ihre Beeinflussungsversuche der Medienagenda auf Themen konzentrieren, die dort ohnehin schon gängig sind. 
Einer der wenigen international-vergleichenden Beiträge (Esmark/Mayerhöffer 20I4), der AkteurInnen aus neun Ländern in Hinblick auf die Präferenz einer medialen oder politischen Handlungslogik in den Blick nimmt, ergibt, dass beim Public-Agenda-Setting Strategien, die die AutorInnen der Medienlogik zuordnen, am häufigsten genannt werden. Allerdings kann diese Untersuchung nichts über inter-individuelle Unterschiede hinsichtlich der Präferenz bzw. Aneignung von Kommunikationsstrategien aussagen.

\section{Forschungslücke: Einflüsse auf individuelle Kommunikationsstrategien}

Gerade in Bezug auf die Gruppe der PolitikerInnen stellt sich folglich die Frage, inwieweit individuelle Merkmale wie Parteizugehörigkeiten und persönliche Wahrnehmungen eine Rolle für ihre Anpassung an die Medienlogik spielen. Generell gibt es erst erstaunlich wenig Erkenntnisse darüber, wie stark politische AkteurInnen der nationalen Ebene bereit sind bzw. sich gezwungen sehen, ihre Kommunikationsstrategie der Medienlogik anzupassen. Es scheint dabei sinnvoll zu sein, auch für sie arbeitende PR-Treibende in die Betrachtung mit einzubeziehen, verfolgen diese doch "mittelbar" die intentionalen Ziele der PolitikerInnen (Jarren/Donges 20II, 169). In der folgenden Analyse stehen folglich zwei Forschungsfragen im Mittelpunkt:

Welches Repertoire an politischen Kommunikationsstrategien weisen PolitikerInnen und PR-Treibende, die in politischen Organisationen arbeiten, in Deutschland und Österreich auf und wie präsent sind darin medienlogikorientierte Strategien? (FF I)

Welche Merkmale von PolitikerInnen und PR-Treibenden erhöhen die Chance, dass sie ihr Kommunikationsverhalten an die Medienlogik anpassen? Welche Rolle spielen insbesondere die Parteizugehörigkeit und subjektive Wahrnehmungen zum Einfluss von Medien und Parlament auföffentliche Diskurse? (FF 2)

Zum Einfluss von subjektiven Wahrnehmungen und Parteizugehörigkeit lassen sich allerdings einige Vermutungen formulieren: Zunächst erscheint es naheliegend, dass die Wahrnehmungen von PolitikerInnen hinsichtlich des politischen Einflusses der Medien für ihre Anpassungsneigung eine Rolle spielen. Denn wie Menschen subjektiv ihre Situation perzipieren, beeinflusst, welche Handlungsmöglichkeiten sie als effektiv zur Erreichung von Zielen ansehen (McLeod/Wise/Perryman 2017). In Bezug auf die öffentliche Debatte über politische Themen ergibt sich daraus, dass PolitikerInnen bzw. PR-Treibende sich umso stärker an der Medienlogik ausrichten müssten, desto eher sie glauben, die Medien bestimmten die politische Agenda (H Ia). Diese Überzeugung dürfte Anreize schaffen oder verstärken, sich mit der Funktionslogik der Medien zu beschäftigen und diese bei der Planung von Botschaften zu beachten. Die Hypothese schließt auch an die Vermutung an, dass die Medienlogik besonders deshalb realen Einfluss in der politischen Kommunikation entfaltet, weil Medien als einflussreich wahrgenommen werden (vgl. z.B. Reinemann 20IO).

Ebenfalls naheliegend ist, dass politische AkteurInnen ihre Kommunikationsstrategien stärker an die Medienlogik anpassen, wenn sie das Vertrauen in parlamentarische Kommunikation als Mittel zum Einbringen von Themen und Standpunkten in die öffentliche Debatte verloren haben. Dann substituiert die mediale Öffentlichkeit gleichsam das Parlament und gewinnt somit zusätzlich an Gewicht, wodurch wiederum die Anreize zur Anpassung steigen. Daher: Je weniger politische AkteurInnen glauben, Kommunikation im Parlament sei effektiv, desto stärker passen sie sich der Medienlogik an (H Ib).

Des Weiteren könnten die Unterschiede zwischen nationalen Kontexten, welche die strukturellen Rahmenbedingungen politischer Kommunikation mitprägen, einflussreich für medienlogikkompatibles Kommunikationsverhalten sein. Wir vergleichen diesbezüglich mit Deutschland und Österreich zwei Parteiendemokratien, die im Bereich des politischen Kommunikationssystems grundsätzlich als ähnlich klassifiziert werden (Hallin/Mancini 2004, Brüggemann/Engesser/ Büchel/Humprecht/Castro 20I4; Pfetsch/Mayerhöffer/ Moring 20I4). Darüber hinaus ist das österreichische Mediensystem im Vergleich zum deutschen im Bereich der Presse jedoch stärker durch Boulevard- und Gratiszeitungen geprägt und im Bereich des audiovisuellen Sektors stärker durch Unterhaltung (Magin/Stark 2015). Befragungen politischer Mandatsträger haben darüber hinaus gezeigt, dass die größte Boulevardzeitung und der öffentlich-rechtliche Sender ORF - dem ebenfalls ein starker Fokus auf unterhaltende Programme attestiert wurde (Wölke 20I2) - aus ihrer subjektiven Sicht mit Abstand die größte Rolle für die Politik spielen (Tenscher 2012). Somit entsteht in Österreich ein stärkerer Wettbewerb um die Platzierung politischer Botschaften bei gleichzeitiger Dominanz von Medien, die entweder durch die Fernsehlogik oder den Stil der Boulevardpresse geprägt sind.

Deshalb vermuten wir, dass der österreichische Kontext zu einer stärker medienlogikorientierten Denkweise bei politischen AkteurInnen beiträgt ( $\mathrm{H}_{2}$ ).

Abgesehen vom Land müssten auch die Parteien als Organisationskontext prägende Strukturen des Kommunikationsverhaltens der AkteurInnen bzw. ihrer Perzeptionen sein. Bisher hat aber keine Untersuchung zeigen können, ob Parteiunterschiede die Einstellung zur Medienlogik in der politischen Kommunikation beeinflussen. Eine wichtige Rolle könnte hier der Unterschied zwischen Parteien, die an der Regierung beteiligt sind und somit Positionen der Exekutive besetzen, und der Opposition spielen. Da Oppositionsparteien am Treffen allgemeinverbindlicher politischer Entscheidungen 
meist nicht direkt beteiligt sind, dient ihnen dabei öffentliche Kommunikation noch stärker als das Mittel, um die politische Tagesordnung zu beeinflussen und für sich günstig zu gestalten. Sie orientieren sich dabei stärker als Regierungsparteien an der Medienagenda (Walgrave/Van Aelst 2006), weil sie oft nur in Kooperation mit Medien so viel Druck auf die Regierung aufbauen können, dass diese sich zu einem Thema äußern muss, sodass es nachhaltig in der politischen Debatte bleibt.

Aus diesen Überlegungen ergibt sich die Vermutung, dass politische AkteurInnen von Oppositionsparteien sich stärker der Medienlogik anpassen als VertreterInnen von Regierungsparteien ( $\mathrm{H}_{3}$ ).

\section{Methodisches Vorgehen}

\subsection{Auswahl der Untersuchungseinheiten und Codierung}

Die Daten der Untersuchung basieren auf einer Sekundäranalyse von Daten aus dem Forschungsprojekt "Political Communication Cultures in Western Europe. A Comparative Study." (für eine Dokumentation der Ergebnisse siehe Pfetsch 20I4; für die Datenbasis des Projekts siehe Pfetsch et al. 20I4). Das vorliegende Untersuchungsmaterial stellt die Antworten auf zwei offene Fragen von insgesamt 318 PolitikerInnen und politischen PR-Treibenden aus Deutschland und Österreich dar, die zusammenfassend in dieser Untersuchung als politische AkteurInnen bezeichnet werden. Sie wurden über einen Positionsansatz ausgewählt, d.h. ausgehend von politischen AkteurInnen in herausgehobenen Positionen wurden top down rangniedrigere EntscheidungsträgerInnen der Institutionen Regierung, Parlament und Parteien befragt (siehe Maurer/Vähämaa 20I4). Gemessen an den angeschriebenen Personen (erreichbare Grundgesamtheit) lag die Ausschöpfung in Deutschland bei den PolitikerInnen bei 25 Prozent und den PR-Treibenden bei 58 Prozent. Die Werte für Österreich waren 2I bzw. 45 Prozent. Der Befragungsmodus bestand aus einer Kombination von telefonischen, schriftlichen und persönlichen Face-to-Face Interviews. Bei der ersten offenen Frage sollten PolitikerInnen und PR-Treibende angeben, ob ihnen über vier in einer vorangestellten, geschlossenen Frage abgefragte Strategien ${ }^{2}$ hinaus Aktivitäten einfallen, „die besonders wirkungsvoll sind, wenn PolitikerInnen öffentliche Aufmerksamkeit er-

2 „PolitikerInnen versuchen auf unterschiedlichsten Wegen, öffentliche Aufmerksamkeit für ihre Anliegen zu wecken. Aus Ihrer Sicht als PolitikerIn, für wie wirkungsvoll halten Sie diese grundsätzlich. Verwenden Sie bitte eine Skala von I = "überhaupt nicht wirkungsvoll” bis 5 = "sehr wirkungsvoll”: „Eine Rede im Parlament“, „Auftritte von Politikern in Talk-Shows“, „Politiker spielen ausgewählten Journalisten gezielt Informationen zu“, „Politiker stellen politische Themen besonders dramatisch dar". regen wollen ${ }^{\text {“ } 3}$ und diese stichwortartig beschreiben. In der zweiten, weiter hinten im Fragebogen platzierten offenen Frage wurde erfragt, was PolitikerInnen tun müssen, „um neue Themen erfolgreich in die öffentliche Diskussion zu bringen“. Dies sollten sie wiederum stichwortartig beschreiben.

Insgesamt gaben die 318 Befragten $(\mathrm{N}=318$; vgl. Tabellen 2-5) 259 verwertbare Antworten. Da die Antworten in Sätzen oder mehreren Stichpunkten gegeben wurden, die mehrere inhaltliche Aspekte enthalten konnten, wurden sie mittels eines grammatikalischen Schemas zerlegt. So konnten 592 syntaktisch-semantische Einheiten gewonnen werden, die dann die Codiereinheiten bildeten. Das Kategoriensystem wurde induktiv entwickelt. Im ersten Schritt wurde das gesamte Material durchgelesen, um ein Gefühl für den Inhalt zu bekommen. Im nächsten Schritt wurde die Hälfte der 592 Codiereinheiten, also rund 300 semantische Einheiten, paraphrasiert, wobei durch eine Zufallsauswahl sichergestellt wurde, dass alle Berufsgruppen und Länder gleichermaßen abgebildet wurden. Danach wurden deckungsgleiche Paraphrasen gestrichen und verwandte Paraphrasen zu einer Kategorie zusammengefasst.

Das Vorgehen soll an den folgenden zwei Beispielen verdeutlicht werden: Die Antwort, „Privatleben des Politikers einsetzen“ wurde mit „Privatleben“ paraphrasiert und im nächsten Schritt - mit Blick auf ähnliche Paraphrasen - zur Kategorie „Personalisierung“ verdichtet. Die Antwort "die außerparlamentarische Bewegung aktivieren, z.B. die Betroffenen-Verbände o.ä." wurde mit „Bündnisse“ paraphrasiert und mit anderen Paraphrasen zur Kategorie "Vernetzung und Koalitionsbildung“ zusammengefasst. Das daraus resultierende vorläufige Kategoriensystem wurde in einer Rückkoppelungsschleife nochmals am bereits analysierten Material geprüft und substanziell ergänzt und verfeinert. Dadurch wurde eine bessere Anpassung der Kategorien an das Material erreicht. In seiner endgültigen Form enthält das Schema insgesamt 25 Kategorien. Tabelle I gibt einen Überblick über diese Kategorien und führt als Ankerbeispiele je eine solche Antwort an, wie sie als prototypisch für die jeweilige Einzelkategorie begriffen wurde.

Das gesamte Datenmaterial, also alle 592 Codiereinheiten, wurde von zwei CodiererInnen anhand der gewonnenen 25 Kategorien quantitativ verschlüsselt. Die Intercoder-Reliabilität wurde mit Krippendorffs Alpha berechnet (Hayes/Krippendorf 2015), das mit Al$p h a=, 75$ einen guten Wert erreichte, gerade angesichts

3 Für eine deduktive Analyse dieser Frage siehe Esmark und Mayerhöffer (2OI4). In Abgrenzung zum hier realisierten induktiven Vorgehen, das unter Hinzunahme einer erweiterten Datenbasis eine breitere Ebene individueller Kommunikationsstrategien adressiert, verfolgt deren Beitrag mit einer Fokussierung auf reines Agenda Setting und der rein theoriebasierten exklusiven Unterscheidung zwischen Medienlogik und politischer Logik ein originär anderes Vorgehen. 
der Komplexität des Kategoriensystems (Früh 2OII). Um die Gesamtreliabilität der Codierung noch zusätzlich zu erhöhen, wurden uneindeutige Fälle im Anschluss noch einmal diskutiert, bis es zu einer Einigung kam.

Das angewandte Methodendesign lässt sich zusammenfassend als „Partially Mixed Sequential Equal Status Design“ (Leech/Onwuegbuzie 2009, 269) bezeichnen: Die qualitative Verdichtung des Materials folgt einer induktiven Logik und trägt der explorativen ersten Forschungsfrage Rechnung. Dies hat den Vorteil, dass die Analyse Aspekte zutage fördern kann, die einem deduktiven Vorgehen per definitionem verschlossen bleiben und scheint der Analyse kognitiver Konzepte - Stichwort "mentale Medialisierung" (Marcinkowski 2015) - angemessener. Die darauffolgende (sequential) deskriptive und inferenzstatistische Auswertung (vgl. 5.I bzw. 5.2) bleibt analytisch vom qualitativen Teil getrennt (partially mixed) und hat das Vermögen, gemäß der zweiten Forschungsfrage, theoretische Zusammenhänge mit den Befunden konfirmatorisch auf Plausibilität zu prüfen. Durch die gegenseitige Unabdingbarkeit der qualitativen und quantitativen Analyse lassen sich diese als gleichberechtigt zur Beantwortung der Forschungsfragen bezeichnen (equal status).

Tabelle 1: Oberkategorien, Einzelstrategien und Ankerbeispiele

\begin{tabular}{|c|c|c|}
\hline Oberkategorie & Einzelstrategie & Ankerbeispiel \\
\hline \multirow{5}{*}{$\begin{array}{l}\text { (1) Erhöhung der } \\
\text { Kompatibilität der } \\
\text { Botschaft mit der } \\
\text { Medienlogik }\end{array}$} & Konflikte und Skandale & „Konfliktinszenierung“ \\
\hline & Personalisierung & „Privatleben“ \\
\hline & Dramatisierung & „Thema dramatisieren“ \\
\hline & Visualisierung & „Bilder produzieren“ \\
\hline & Inszenieren von Ereignissen & „symbolträchtige Aktionen“ \\
\hline \multirow{5}{*}{$\begin{array}{l}\text { (2) Gezielte } \\
\text { Medienansprache }\end{array}$} & $\begin{array}{l}\text { Unbestimmte Medienansprache wie Pressearbeit } \\
\text { und Medienkooperationen }\end{array}$ & „eigene Pressekonferenz“ \\
\hline & Eigene Medienbeiträge & $\begin{array}{l}\text { "Grundsatzbeiträge in den Medien } \\
\text { (Positionspapiere)“ }\end{array}$ \\
\hline & Ansprache bestimmter Medien & „Talkshows“ \\
\hline & Exklusivität bei der Kooperation mit Medien & "gezielte Exklusivgeschichten“ \\
\hline & Weitere PR-Maßnahmen, Marketing, Kampagnen & „Werbematerial“ \\
\hline \multirow{2}{*}{$\begin{array}{l}\text { (3) Kooperation } \\
\text { mit bestimmten } \\
\text { JournalistInnen }\end{array}$} & Persönlicher Kontakt mit JournalistInnen & $\begin{array}{l}\text { „einen Journalisten überzeugen und gemeinsam } \\
\text { eine Strategie entwickeln“ }\end{array}$ \\
\hline & Kooperation mit JournalistInnen & „mit einem Medium zusammen arbeiten“ \\
\hline \multirow{5}{*}{$\begin{array}{l}\text { (4) Suche nach } \\
\text { Verbündeten und direkte } \\
\text { Kommunikation mit } \\
\text { BürgerInnen }\end{array}$} & Vernetzung \& Koalitionsbildung & „unerwartete Bündnisse“ \\
\hline & Einsatz politischer Instrumente und Strategien & „Misstrauensanträge“ \\
\hline & Dialog mit der Bevölkerung & „Vertrauensarbeit vor Ort“ \\
\hline & Betroffenheit schaffen & $\begin{array}{l}\text { „persönliche Schicksale und Ereignisse (Fall } \\
\text { Arigona)“ }\end{array}$ \\
\hline & Öffentliche Meinung antizipieren (Demoskopie) & „Mainstream erkennen“ \\
\hline \multirow{5}{*}{$\begin{array}{l}\text { (5) Überzeugen durch } \\
\text { sachliche Argumentation } \\
\text { und Fakten }\end{array}$} & Sprache, Rhetorik und Argumentation & „Inhalte verständlich erklären“ \\
\hline & Authentizität und Glaubwürdigkeit & „inhaltlich authentisch darlegen“ \\
\hline & $\begin{array}{l}\text { Überzeugung durch Fakten, Sachlichkeit und } \\
\text { Beispiele }\end{array}$ & „faktengestützte Information“ \\
\hline & Lösungen aufzeigen & „Lösungs- oder Kritikmöglichkeiten kommunizieren“ \\
\hline & Neuigkeitswert der Botschaft & „neue Aspekte auf die Agenda setzen“ \\
\hline \multirow{3}{*}{ Weitere Strategien } & Timing & „richtiger Zeitpunkt“ \\
\hline & Ausdauer & $\begin{array}{l}\text { „über längere Zeit hinweg durchhalten und am } \\
\text { Leben halten“ }\end{array}$ \\
\hline & $\begin{array}{l}\text { Eigenschaften/Image von KommunikatorInnen } \\
\text { nutzen }\end{array}$ & „Popularität des Politikers ist bedeutsam“ \\
\hline
\end{tabular}




\subsection{Auswertung}

Vor der Auswertung wurden 22 der 25 Kategorien, in welche die Einzelstrategien differenziert wurden, zu fünf Oberkategorien zusammengefasst: (I) Erhöhung der Kompatibilität der Botschaft mit der Medienlogik, (2) gezielte Medienansprache, (3) Kooperation mit bestimmten JournalistInnen, (4) Suche nach Verbündeten und direkte Kommunikation mit BürgerInnen, (5) Überzeugen durch sachliche Argumentation und Fakten (siehe Tabelle I). Zu diesen Strategien wurden im ersten Schritt der Auswertung Aggregatvergleiche auf Länderund Parteiebene durchgeführt.

Im zweiten Schritt der Auswertung ging es darum zu prüfen, welche Variablen die Wahl bzw. Nicht-Wahl insbesondere medienlogischer Strategien bei einzelnen politischen AkteurInnen erklären können. Dabei berücksichtigen wir außer Land und Parteizugehörigkeit die individuelle Medieneinflusswahrnehmung sowie das Vertrauen eines Befragten in parlamentarische Kommunikation. Zur Ermittlung des Ländereinflusses wurde ein Dummy für Österreich konstruiert. Zur Ermittlung des Parteieinflusses wurden die Parteien aus Gründen der Vergleichbarkeit zunächst den europäischen Parteifamilien - sozialistische (Linke), grüne (jeweils die Grünen), sozialdemokratische (SPD, SPÖ), liberale (FDP), konservative (CDU/CSU, ÖVP), rechtspopulistische Parteien (FPÖ) - zugeordnet. Dann wurde auch hier eine Dummy-Kodierung durchgeführt, sodass die konservativen Parteien (CDU/CSU und ÖVP) die Referenzkategorie bildeten.

Darüber hinaus wurde eine Frage aus demselben Fragebogen, die Medieneinflusswahrnehmung standardisiert misst, verwendet. Sie bezog sich auf die politische Agenda-Setting-Macht der Medien und lautete: „Die Medien und nicht politische Akteure entscheiden, welche Themen in der Politik wichtig sind und die politische Agenda bestimmen." Die Antwortmöglichkeiten bewegten sich zwischen starker Ablehnung (I) und starker Zustimmung (5) zu dieser Aussage. Das Vertrauen in die Wirksamkeit von öffentlicher Kommunikation im Parlament wurde schließlich mit folgender Aussage ermittelt: „Politiker und Politikerinnen versuchen auf unterschiedlichsten Wegen, öffentliche Aufmerksamkeit für ihre Anliegen zu wecken. Für wie wirkungsvoll halten Sie eine Rede im Parlament?" Antwortmöglichkeiten bewegten sich zwischen I (überhaupt nicht wirkungsvoll) und 5 (sehr wirkungsvoll). Da die abhängige Variable die Nennung (I) oder Nicht-Nennung (o) einer bestimmten Kommunikationsstrategie in einer der offenen Fragen war, wurden binäre logistische Regressionen durchgeführt.

Tabelle 2: Bewertung der Effektivität von Aufmerksamkeitsstrategien in Prozent der Befragten nach Land und Berufsrolle (geschlossene Abfrage)

\begin{tabular}{|c|c|c|c|c|c|c|c|c|c|c|c|c|}
\hline & \multicolumn{3}{|c|}{$\begin{array}{l}\text { Eine Rede im Parlament } \\
\text { ist ... }\end{array}$} & \multicolumn{3}{|c|}{$\begin{array}{l}\text { Auftritte in Talkshows } \\
\text { sind ... }\end{array}$} & \multicolumn{3}{|c|}{$\begin{array}{c}\text { Informationen } \\
\text { „durchzustechen“ ist ... }\end{array}$} & \multicolumn{3}{|c|}{$\begin{array}{l}\text { Themen zu dramati- } \\
\text { sieren und Konflikte } \\
\text { zu betonen ist ... }\end{array}$} \\
\hline & 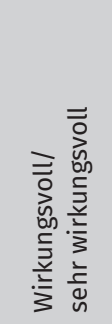 & 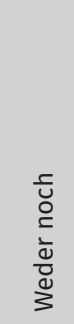 & 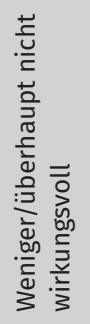 & 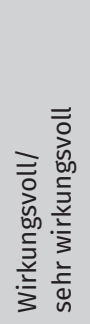 & 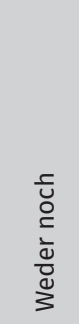 & 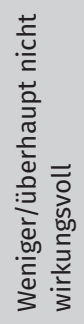 & 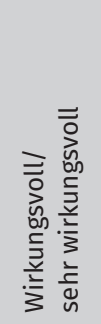 & 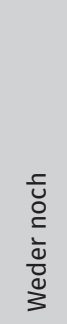 & 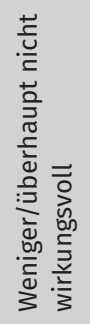 & 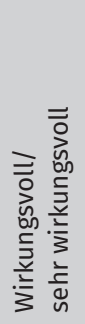 & 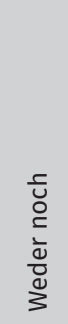 & 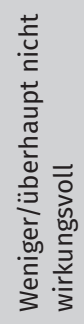 \\
\hline Total & 7,0 & 23,0 & 70,0 & 56,7 & 30,4 & 12,8 & 77,6 & 16,0 & 6,4 & 50,6 & 36,5 & 12,9 \\
\hline Deutschland & 7,1 & 28,8 & 64,1 & 63,1 & 27,5 & 9,4 & 74,3 & 17,5 & 8,2 & 44,7 & 39,4 & 15,9 \\
\hline Österreich & 7,0 & 16,1 & 77,0 & 48,9 & 34,0 & 17,0 & 81,6 & 14,2 & 4,2 & 57,8 & 32,9 & 9,3 \\
\hline PolitikerInnen & 9,1 & 21,4 & 69,5 & 59,7 & 26,9 & 13,4 & 71,3 & 19,7 & 9,1 & 48,9 & 36,0 & 15,0 \\
\hline BeraterInnen & 4,0 & 25,4 & 70,6 & 52,4 & 35,7 & 11,9 & 87,1 & 10,5 & 2,4 & 53,3 & 37,1 & 9,7 \\
\hline
\end{tabular}

$N=313$ 


\section{Ergebnisse}

Bevor die einer induktiven Logik folgenden Analysen zur Beantwortung der Forschungsfragen dargelegt werden, soll im Folgenden ein kurzer Überblick über die Ergebnisse der deduktiv konzipierten, geschlossenen Abfrage von Aufmerksamkeitsstrategien geleistet werden, die im Fragebogen vorangestellt waren (siehe 5.I) und die Kernanalyse der Untersuchung demnach kontextualisieren (Tabelle 2). Bei der geschlossenen Abfrage halten Befragte die Weitergabe von Informationen mit 78 Prozent Zustimmung für am wirkungsvollsten, um öffentliche Aufmerksamkeit zu erreichen, gefolgt von Auftritten in Talkshows (57\%), Dramatisierung und Konflikthaftigkeit $(51 \%)$ sowie Reden im Parlament (7\%). Aus Perspektive der hier untersuchten Medienlogik kann also festgehalten werden, dass mit Dramatisierung und Konflikthaftigkeit für rund die Hälfte der politischen AkteurInnen eine auf Medienlogik basierende Strategie als effektiv begriffen wird, sie demnach einen zentralen Stellenwert einnimmt. ${ }^{4}$

Einen signifikanten Länderunterschied gibt es hinsichtlich der Konflikt- und Dramatisierungs-Strategie: Sie wird von knapp 58 Prozent der ÖsterreicherInnen für effektiv gehalten, aber nurvon ca. 45 Prozent der Befragten aus Deutschland. 5 Darüber hinaus halten auch signifikant mehr österreichische Befragte Parlamentskommunikation für wirkungslos. ${ }^{6}$ Bezüglich möglicher Berufsgruppenunterschiede zeigen sich ebenfalls kaum signifikante Differenzen. Einzig die Strategie, Informationen "durchzustechen", halten BeraterInnen mit einer Zustimmung von knapp 90 Prozent für signifikant wirkungsvoller ${ }^{7}$ als PolitikerInnen. ${ }^{8}$

4 Forschungsfrage I tangiert dies insofern jedoch wenig, als dass diese nach dem jeweiligen Repertoire, also der Vielfalt und Unterschiedlichkeit von Strategien fragt und damit bewusst den Fokus auf Antworten legt, die den bisherigen Forschungskorpus erweitern; es ist nicht davon auszugehen, dass Befragte in einem verminderten Umfang die Bandbreite ihrer Strategien offenlegen, nachdem ihnen eine Auswahl bereits erforschter Strategien vorgelegt wurde.

$5 \quad \chi^{2}(4,313)=13,65, \mathrm{p}<0,01$

$6 \chi^{2}(4,313)=19,7 \mathrm{I}, \mathrm{p}<0, \mathrm{OI}$

$7 \quad \chi^{2}(4,313)=13,4$ I, $p<0,01$

8 Die relative Homogenität ist zwar als wertvoller erster Hinweis für die komparative Kernanalyse der Untersuchung zu sehen, die vorgelagerte geschlossene Abfrage gefährdet die Gültigkeit von Forschungsfrage 2 der vergleichenden Analyse der offenen Frage, welche nach Länder-, Partei- und Professionsunterschieden fragt, jedoch insofern nicht, als dass es keinerlei Indizien dafür gibt, dass eine dieser Gruppen systematisch stärker oder schwächer von der vorgelagerten Abfrage beeinflusst wird. Die zusätzliche Verwendung von Antworten einer zweiten, an einer späteren Stelle im Fragebogen platzierten offenen Frage, die demnach gar nicht von einem möglichen derartigen Effekt beeinflusst wird, stärkt die Validität zusätzlich.

\subsection{Deskriptive Befunde}

Der Blick auf die Ergebnisse - zunächst für das gesamte Sample - zeigt, dass die politischen AkteurInnen ein breites Spektrum von Kommunikationsstrategien nennen (Tabellen I, 3 und 4). Die meisten Befragten nennen Strategien, die sich der Kategorie ,Überzeugen durch sachliche Argumente und Fakten' zuordnen lassen. Knapp die Hälfte gibt eine solche Strategie zumindest einmalig als Antwort auf eine der beiden Fragen an. Danach folgt jedoch schon die Kategorie ,Medienlogik', die von den einem Drittel der Befragten mindestens einmal genannt wurde. Dahinter liegen die Strategien ,Suche nach Verbündeten/direkte Ansprache der BürgerInnen', gezielte Medienansprache und ,Kooperation mit JournalistInnen: Betrachtet man die Anteile für die einzelnen Strategien, die der Kategorie Medienlogik zugeordnet wurden, genauer, erkennt man, dass die meisten Befragten ,Inszenierung von Ereignissen" nennen. Beinahe ein Drittel hält diese Strategie für effektiv, die darauf abzielt, künstlich eine Bühne zu kreieren, die von Medien gut ausgeleuchtet werden kann. Inhaltliche Tiefe erscheint hierbei nebensächlich für den Erfolg der Kommunikation. Ein Teil der Befragten betont, dass die Ereignisse einen überraschenden, aktionistischen oder spektakulären Charakter besitzen müssen. Gemäß dem primären Ziel, durch Außergewöhnliches die Aufmerksamkeitsschwelle der Medien zu überwinden und damit den gewohnten Informationsfluss politischer Kommunikation zu unterbrechen, kann diese Strategie als disruptiv bezeichnet werden. Sie entspricht dem von Altheide $(2014,2)$ genannten Aspekt der Unmittelbarkeit medienlogischer Kommunikation, der zulasten der Vermittlung von Hintergründen geht. Innerhalb der Gruppe medienlogischer Strategien werden darüber hinaus Skandalisierung und das Herausstellen (innerparteilicher) Konflikte häufig genannt.

Wir konzentrieren uns im Weiteren darauf, welche Gruppen besonders häufig medienlogikorientierte Kommunikationsstrategien nennen. Der Vergleich zwischen den Parteien zeigt, dass die Grünen deutlich am häufigsten mit der Medienlogik kompatible Strategien nennen, allerdings zeigt ein Chi-Quadrat Test keine signifikanten Unterschiede im Vergleich aller Parteien. ${ }^{9}$ Einen höheren Wert als die zum Zeitpunkt der Datenerhebung in der Regierung befindlichen Parteien (in beiden Ländern Sozialdemokraten und Konservative) erreicht auch die Oppositionspartei FDP. Allerdings sind die Anteile bei Vertretern der ebenfalls oppositionellen Linken und FPÖ geringer als die der regierenden Parteien (CDU/CSU, SPD, SPÖ, ÖVP). Auch ein Vergleich der Regierungs- und Oppositionsparteien erbringt keinen

$9 \chi^{2}(5)=9, \mathrm{IO}, p=0, \mathrm{IO} 5$ 
Tabelle 3: Nennung von Aufmerksamkeitsstrategien in Prozent (in Fallzahlen) der Befragten nach Land, Berufsrolle und Partei

\begin{tabular}{|c|c|c|c|c|c|c|c|c|c|c|}
\hline \multirow[b]{2}{*}{ Land } & \multicolumn{2}{|c|}{ Medienlogik } & \multicolumn{2}{|c|}{ Medienansprache } & \multicolumn{2}{|c|}{$\begin{array}{l}\text { Kooperation mit } \\
\text { JournalistInnen }\end{array}$} & \multicolumn{2}{|c|}{$\begin{array}{l}\text { Verbündete } \\
\text { und direkte } \\
\text { Kommunikation } \\
\text { mit BürgerInnen }\end{array}$} & \multicolumn{2}{|c|}{$\begin{array}{c}\text { Überzeugen } \\
\text { durch Fakten und } \\
\text { Rhetorik }\end{array}$} \\
\hline & & & & & & & & & & \\
\hline Deutschland & 30,1 & (52) & 30,1 & $(52)$ & 7,5 & (13) & 29,5 & (51) & 41,6 & (72) \\
\hline Österreich & 37,9 & (55) & 16,6 & (24) & 13,1 & (19) & 29,0 & $(42)$ & 45,5 & (66) \\
\hline Total & 33,6 & (107) & 23,9 & (76) & 10,1 & (32) & 29,2 & (93) & 43,4 & (138) \\
\hline \multicolumn{11}{|l|}{ Berufsrolle } \\
\hline PolitikerInnen & 33,2 & (63) & 17,9 & (34) & 10,5 & (20) & 28,4 & (54) & 41,1 & (78) \\
\hline BeraterInnen & 34,4 & (44) & 32,8 & $(42)$ & 9,4 & $(12)$ & 30,5 & (39) & 46,9 & (60) \\
\hline Total & 33,6 & (107) & 23,9 & $(76)$ & 10,1 & (32) & 29,2 & (93) & 43,4 & (138) \\
\hline \multicolumn{11}{|l|}{ Partei } \\
\hline Linke & 10,0 & (2) & 40,0 & (8) & 5,0 & (1) & 45,0 & (9) & 35,0 & (7) \\
\hline Grüne & 48,3 & (14) & 13,8 & (4) & 17,2 & (5) & 24,1 & (7) & 34,5 & (10) \\
\hline Sozialdemokratische & 37,0 & (27) & 19,2 & $(14)$ & 9,6 & (7) & 30,1 & $(22)$ & 47,9 & (35) \\
\hline Liberale & 42,9 & (6) & 42,9 & (6) & 7,1 & (1) & 14,3 & $(2)$ & 57,1 & (8) \\
\hline Konservative Volkspartei & 32,2 & (28) & 16,1 & $(14)$ & 11,5 & $(10)$ & 28,7 & $(25)$ & 36,8 & (32) \\
\hline Rechtspopulistische & 25,0 & (3) & 25,0 & (3) & 8,3 & (1) & 16,7 & $(2)$ & 33,3 & (4) \\
\hline Total & 34,0 & (80) & 20,9 & (49) & 10,6 & $(25)$ & 28,5 & $(67)$ & 40,9 & (96) \\
\hline
\end{tabular}

N (Länder- und Berufsrollenvergleich $)=318, \mathrm{~N}($ Parteivergleich $)=235$

signifikanten Unterschied. ${ }^{10}$ Somit bestätigt die bivariate Analyse die Vermutung, die Medienlogik habe generell eine größere Bedeutung für Oppositionsparteien ( $\mathrm{H}$ 3), zunächst zwar nicht. Es bleibt jedoch zu prüfen, ob einzelne Oppositionsparteien stärker medienlogische Strategien anwenden, wenn gleichzeitig für andere Einflussfaktoren kontrolliert wird (Tabelle 5).

Betrachtet man die einzelnen medienlogikkompatiblen Strategien, dann zeigt sich ein erstaunlicher Gleichklang zwischen den Ländern. Die jeweiligen Anteile der Befragten, die jeweils bestimmte Strategien nennen, unterscheiden sich nur um wenige Prozentpunkte. Der einzige signifikante Unterschied zeigt sich bei der Strategie ,Dramatisierung', die I4,5 Prozent der österreichischen aber nur 5,2 Prozent der deutschen Befragten nennen. ${ }^{\text {II }}$ Dieser Unterschied deutete sich schon in der geschlossenen Frage an. Bei der Orientierung von

IO $\chi^{2}(\mathrm{I})=0,25, p=0,875$

II $\chi^{2}(\mathrm{I})=7,95, p<$ O,OI
Kommunikationsstrategien an der Medienlogik zeigen sich aber insgesamt nur schwach ausgeprägte Länderunterschiede. Dass Medienlogik insgesamt eine etwas größere Rolle in Österreich spielt, entspricht tendenziell jedoch $\mathrm{H} 2$.

\subsection{Multivariate Analyse}

Eine bivariate Betrachtung kann nur erste Anhaltspunkte dafür liefern, in welchem Ausmaß Partei und Land Einfluss auf die Anpassung der AkteurInnen an medienlogikorientierte Kommunikationsstrategien haben. Wir haben hier vermutet, dass neben Land und Parteizugehörigkeit die subjektive Medieneinflusswahrnehmung ( $\mathrm{H}$ Ia) und das Vertrauen in die Wirksamkeit parlamentarischer Kommunikation ( $\mathrm{H} \mathrm{Ib}$ ) die Einstellungen $\mathrm{zu}$ Kommunikationsstrategien ebenfalls beeinflussen müssten. Darüber hinaus könnten sich natürlich auch sozio-demografische Variablen wie Alter, Berufserfahrung und Berufsrolle darauf auswirken. Daraus ergibt 
Tabelle 4: Nennung von Strategien der Medienlogik in Prozent (in Fallzahlen) der Befragten nach Land, Berufsrolle und Partei

\begin{tabular}{|c|c|c|c|c|c|c|c|c|c|c|}
\hline \multirow[b]{2}{*}{ Land } & \multicolumn{2}{|c|}{$\begin{array}{c}\text { Konflikte } \\
\text { und Skandale }\end{array}$} & \multicolumn{2}{|c|}{ Dramatisierung } & \multicolumn{2}{|c|}{ Personalisierung } & \multicolumn{2}{|c|}{ Visualisierung } & \multicolumn{2}{|c|}{$\begin{array}{l}\text { Inszenierung von } \\
\text { Ereignissen }\end{array}$} \\
\hline & & & & & & & & & & \\
\hline Deutschland & 22,0 & (38) & 5,2 & (9) & 7,5 & (13) & 6,4 & (11) & 30,1 & (52) \\
\hline Österreich & 25,5 & (37) & 14,5 & (21) & 7,6 & (11) & 6,2 & (9) & 35,9 & (52) \\
\hline Total & 23,6 & (75) & 9,4 & (30) & 7,5 & (24) & 6,3 & (20) & 32,7 & (104) \\
\hline \multicolumn{11}{|l|}{ Berufsrolle } \\
\hline PolitikerInnen & 24,2 & (46) & 7,9 & (15) & 6,8 & (13) & 4,7 & (9) & 30,5 & (58) \\
\hline BeraterInnen & 22,7 & (29) & 11,7 & (15) & 8,6 & $(11)$ & 8,6 & (11) & 35,9 & (46) \\
\hline Total & 23,6 & (75) & 9,4 & (30) & 7,5 & (24) & 6,3 & (20) & 32,7 & (104) \\
\hline \multicolumn{11}{|l|}{ Partei } \\
\hline Linke & 15,0 & (3) & 0,0 & $(0)$ & 5,0 & (1) & 0,0 & $(0)$ & 30,0 & (6) \\
\hline Grüne & 27,6 & (8) & 20,7 & (6) & 3,4 & (1) & 17,2 & (5) & 41,4 & (12) \\
\hline Sozialdemokratische & 24,7 & (18) & 6,8 & (5) & 12,3 & (9) & 4,1 & (3) & 30,1 & (22) \\
\hline Liberale & 28,6 & (4) & 0,0 & (0) & 7,1 & (1) & 21,4 & (3) & 42,9 & (6) \\
\hline Konservative Volkspartei & 23,0 & (20) & 13,8 & (12) & 5,7 & (5) & 5,7 & (5) & 27,6 & (24) \\
\hline Rechtspopulistische & 8,3 & (1) & 0,0 & $(0)$ & 0,0 & (0) & 0,0 & $(0)$ & 33,3 & (4) \\
\hline Total & 23,0 & (54) & 9,8 & (23) & 7,2 & (17) & 6,8 & (16) & 31,5 & (74) \\
\hline
\end{tabular}

$N($ Länder- und Berufsrollenvergleich $)=318, N$ (Parteivergleich $)=235$

sich, dass für alle diese Variablen kontrolliert werden muss. Deshalb werden im nächsten Schritt die Ergebnisse einer logistischen Regression auf die Angabe/NichtAngabe medienlogischer Kommunikationsstrategien als effektives Mittel der politischen Kommunikation dargestellt (Tabelle 5). Haben Befragte in den offenen Fragen eine solche Strategie genannt, wurde dies jeweils mit I codiert, andernfalls mit o.

Die Variablen Land, Partei, subjektive Medieneinflusswahrnehmung und Vertrauen in parlamentarische Kommunikation bilden die zentralen unabhängigen Variablen des Modells. Geschlecht, Berufsrolle und Alter der Befragten bilden die Kontrollvariablen. Die Parteizugehörigkeit der Befragten wurde in sechs Indikatorvariablen umcodiert, wobei konservative Parteien (CDU/CSU, ÖVP) die Referenzgruppe bilden. In Bezug auf die Variablen Geschlecht, Berufsrolle und Land bildeten Frauen, PolitikerInnen und Deutschland die Referenzgruppen.

Wie aus Tabelle 5 ersichtlich, erweist sich das Gesamtmodell als signifikant und weist ein zufrieden- stellendes Pseudo- $R^{2}$ von o,II9 auf. Mit Blick auf die Parteizugehörigkeit offenbart sich nur ein positiver signifikanter Effekt, nämlich der einer Zugehörigkeit zu den Grünen. Ist ein Befragter bei den Grünen, erhöht sich folglich die Wahrscheinlichkeit, eine medienlogische Strategie anzugeben. Der Effektkoeffizient ergibt einen Wert von 2,7I, was bedeutet, dass die Zugehörigkeit eines Befragten zu dieser Partei die Odds der abhängigen Variable im Vergleich zu Mitgliedern konservativer Volksparteien (Referenzgruppe) um I70 Prozent erhöht. Bemerkenswert ist, dass dieser Effekt auch bei der Kontrolle einer großen Zahl konkurrierender Einflussfaktoren besteht. Am Rande sei angemerkt, dass sich die Odds auch bei Zugehörigkeit zur FDP stark erhöhen, allerdings ist dieser Effekt aufgrund zu geringer Fallzahlen nicht signifikant. Im Gegensatz dazu hat die Effektivitätsbewertung parlamentarischer Kommunikation einen signifikant negativen Einfluss. Wenn sich das Vertrauen in die Effektivität parlamentarischer Kommunikation um einen Skalenpunkt erhöht, sin- 
Tabelle 5: Einflüsse auf die Nennung medienlogischer Kommunikationsstrategien als Mittel des Agenda-Settings (logistische Regressionen)

\section{$\operatorname{Exp}(\mathrm{bj})$}

\begin{tabular}{lr}
\hline Partei (Referenz: Konservative Volkspartei: CDU/CSU, ÖVP) & 0,31 \\
$\quad$ Linke & $2,71^{*}$ \\
Grüne & 1,37 \\
$\quad$ Sozialdemokratische & 2,04 \\
$\quad$ Liberale & 0,88 \\
$\quad$ Rechtspopulistische & 1,25 \\
Land (Referenz: Deutschland) & 1,23 \\
Medien haben großen Einfluss auf politische Tagesordnung & \\
(1= trifft nicht zu, 5 = trifft zu) & $0,56^{* *}$ \\
Effektivität von Parlamentsreden (1 = niedrig, 5 = hoch) & 1,00 \\
Berufsrolle (Referenz: Politikerlnnen) & 1,49 \\
Geschlecht (Referenz: Frauen) & 1,01 \\
Alter & 0,34 \\
Konstante & $0,12^{*}$ \\
Pseudo-Rº (Nagelkerke) & \\
\hline &
\end{tabular}

$N=214\left({ }^{*}: p \leq, 05,{ }^{* *}: p \leq, 01,{ }^{* * *}: p \leq, 001\right)$

ken die Odds der abhängigen Variable um 44 Prozent. Umgekehrt bedeutet dies, dass Personen mit geringem Vertrauen in parlamentarische Kommunikation eine größere Chance haben, medienlogikorientierte Strategien zu nennen. Darüber hinaus haben weder Land, noch Alter, Berufsrolle oder Geschlecht einen systematischen Einfluss.

Zusammenfassend ergibt sich damit folgendes Fazit für unsere Hypothesen: Entgegen $\mathrm{H}$ Ia hat die Wahrnehmung, Medien seien politisch sehr einflussreich, keinen Einfluss auf die Neigung, sich an die Medienlogik anzupassen. Allerdings bestätigt sich $\mathrm{H}$ Ib, da die Überzeugung, Parlamentskommunikation sei ein ineffektives Mittel zum Einbringen von Themen in die politische Öffentlichkeit, wie vermutet zu einem Anstieg der Wahrscheinlichkeit führt, Medienlogik als effektiv zu erachten. Während es für die Anpassung egal zu sein scheint, ob die Befragten im deutschen oder österreichischen Kontext mit Medien interagieren ( $\mathrm{H} 2$ also widerlegt wird), können wir die dritte Hypothese nur eingeschränkt bestätigen: Die Zugehörigkeit zu bestimmten Oppositionsparteien - hier vor allem den Grünen - steigert tendenziell die Neigung, sich der Medienlogik anzupassen.

\section{Diskussion}

Das primäre Ziel der vorliegenden Untersuchung war es, herauszufinden, wie stark politische AkteurInnen ihre individuellen politischen Kommunikationsstrategien der Medienlogik anpassen. Dazu wurde zunächst erörtert, wodurch sich an der Medienlogik orientierte, politische Kommunikation gegenüber anderen Strategien auszeichnet. Dann wurden von politischen AkteurInnen aus Deutschland und Österreich in einer 2008 und 2009 durchgeführten Befragung genannte Kommunikationsstrategien, die sie als effektiv zur Beeinflussung politischer Diskurse ansehen, mit diesen Merkmalen verglichen, um so ihre Anpassung an die Medienlogik zu ermitteln. Es zeigt sich, dass immerhin ein Drittel der 318 Befragten mindestens eine Strategie nannte, die Merkmalen der Medienlogik entspricht - aufgrund der vorgelagerten geschlossenen Abfrage von Aufmerksamkeitsstrategien, bei denen rund die Hälfte mit Dramatisierung und Konflikthaftigkeit eine spezifische medienlogische Strategie für effektiv hält, ist damit gesichert von einem hohen Stellenwert von Medienlogik auszugehen. Weder zwischen den Ländern, noch zwischen den Parteien waren jedoch im Aggregat signifikante Unterschiede feststellbar, wenn man von einem stärkeren Hang zur Dramatisierung und konfliktträchtigen $\mathrm{Zu}$ - 
spitzung in Österreich, der sich sowohl in den offenen als auch den geschlossenen Abfragen zeigte, absieht. Im zweiten Schritt wurde mittels multipler logistischer Regression auf individueller Ebene bestimmt, wodurch die (Nicht-)Nennung medienlogikkompatibler Strategien beeinflusst wird. Diese Analyse ergab, dass vor allem die Zugehörigkeit zu einer grünen Partei und die Einschätzung, Kommunikation im Parlament sei ineffektiv, die Wahl medienlogischer Strategien begünstigen. Keinen Effekt hatte dagegen die Wahrnehmung eines starken Medieneinflusses auf die politische Themenagenda, das Geschlecht, das Alter und die Berufsrolle der Befragten. Der nationale Kontext machte ebenfalls keinen Unterschied für die Anpassung an die Medienlogik insgesamt.

Die Befunde zum Einfluss der in beiden Ländern oppositionellen Grünen auf die Wahl von Medienlogik stehen in Einklang mit Befunden inhaltsanalytischer Forschung zum Policy-Agenda-Setting, die ebenfalls eine engere Beziehung zwischen Opposition und Medienagenda zeigen als zwischen Mehrheitsparteien und Medienagenda (Walgrave/Van Aelst 2006). Allerdings muss darauf hingewiesen werden, dass die anderen Oppositionsparteien - FDP, Linke, FPÖ - keinen signifikanten Effekt auf die Nennung von Medienlogik-Strategien aufweisen.

Schließlich hat die Untersuchung gezeigt, dass $\mathrm{Me}-$ dienlogik als politische Kommunikationsstrategie von politischen AkteurInnen als stark antithetisch zu klassischer parlamentarischer Kommunikation wahrgenommen wird. Der Glaube, die politische Debatte könnte durch Nutzung der parlamentarischen Bühne beeinflusst werden, verringert die Neigung, Kommunikate medienlogikorientiert gestalten zu wollen und umgekehrt. Dies lässt sich so interpretieren, dass eine Anpassung an die Medienlogik in der Tat mit einer Aushöhlung der Parlamente als Orte kommunikativer Entscheidungslegitimierung einhergeht. Dies ist bemerkenswert, denn Parlamentskommunikation stand und steht ebenfalls im Zentrum der Aufmerksamkeit auch kommunikationswissenschaftlicher Forschung, etwa wenn es um die öffentliche Funktion kleiner Anfragen geht (etwa Kepplinger 2009).

Bei der Interpretation der Ergebnisse muss berücksichtigt werden, dass die Messung von Einstellungen zur Effektivität von Kommunikationsstrategien über offene Fragen Vor- und Nachteile birgt. Ein Nachteil ist, dass sich die Strategien zum Teil auf die Botschaft beziehen und zum Teil auf ihre Adressaten. Das sich aus diesen Verständnisunterschieden der Befragten ergebende breite Spektrum an Antworten spiegelt sich auch in einem breiten Kategorienspektrum wider. Allerdings hat die Verwendung einer offenen Frage auch klare Vorteile. Das offene Format erlaubte es den AkteurInnen, genau das anzugeben, was ihnen selbst am Wichtigsten erschien, ohne durch vorgegebene Antwortalternativen eingeschränkt zu sein. Somit können wir sicher sein, dass solche Nennungen anzeigen, dass den Befragten die genannten Aspekte tatsächlich von sich aus eingefallen sind. Einschränkend müssen an dieser Stelle die im Fragebogen vorangegangenen geschlossenen Fragen zur Effektivität bestimmter Strategien genannt werden, die zwar den Stellenwert der jeweiligen Strategien in der offenen Abfrage vermindern können, die Beantwortung der Forschungsfragen jedoch nicht einschränken (vgl. Fußnoten 4 und 8).

Als mögliche Limitation muss der Erhebungszeitpunkt, der knapp Io Jahre zurückliegt, thematisiert werden, da dies zum einen in Bezug auf eine veränderte politische "Landkarte" und zum anderen bezüglich des fortschreitenden Medienwandels virulent wird. Betreffend Ersterem muss die Übertragbarkeit der Ergebnisse auf mitunter veränderte Kräfteverhältnisse und Ausrichtungen von Parteien reflektiert werden. Darüber hinaus offeriert der zurückliegende Befragungszeitraum jedoch die retrospektiv aufschlussreiche Möglichkeit, die beiden Länder zu einem Zeitpunkt zu vergleichen, zu dem die Opposition (mit der FPÖ in Österreich) in einem Land stark rechtspopulistisch geprägt war, während dies (vor den Erfolgen der AfD in Deutschland) im anderen Land noch nicht der Fall war, was als bedeutsame Reflexionsfolie für die aktuelle politische Lage dienen kann. Außerdem muss der Einfluss des Medienwandels, speziell der gestiegene Stellenwert von Online-Aktivitäten, beachtet werden. Zum Befragungszeitpunkt waren diese im Bereich der politischen Kommunikation noch auf einem weniger bedeutenden Stand.

Diskutiert werden könnte schließlich auch, warum wir Pressearbeit und Kooperation mit JournalistInnen nicht als Anzeichen für eine Anpassung an die Medienlogik werten, sondern als getrennte Kategorien behandeln. Hier argumentieren wir auf Basis des von Strömbäck (2008) überzeugend herausgearbeiteten Unterschiedes zwischen medienvermittelter und mediatisierter Kommunikation: Die genaue Planung, über welche Medien oder mithilfe welcher JournalistInnen kommuniziert wird, zeigt zwar, dass politische AkteurInnen sich der Bedeutung der Medien als Vermittler bewusst sind, dies muss aber nicht heißen, dass die Kommunikationsinhalte selbst so gestaltet werden, dass sie den medialen Selektions- und Darstellungsregeln entsprechen. Für die Diagnose einer Anpassung an die Medienlogik kommt es aber genau darauf an.

Schließlich kann mit diesem Querschnittsdesign ein Kernpunkt der Mediatisierung, nämlich ihr Prozesscharakter (vgl. z.B. Reinemann 20IO), nicht untersucht werden. Es stand allerdings auch nicht im Mittelpunkt unserer Untersuchung, den Nachweis zu erbringen, dass sich bestimmte Kommunikationsstrategien oder Effektivitätswahrnehmungen über die Zeit verändert haben. Vielmehr setzten wir als Prämisse voraus, dass politi- 
sche AkteurInnen auf die manifesten Veränderungen im System der politischen Kommunikation, die hin zu mehr medialer Autonomie gehen, seit geraumer Zeit reagieren und versuchen, sich daran anzupassen (Blumler/Kavanagh, 1999; Kepplinger 2009; Strömbäck/Esser 2014). Allerdings zeigt dieser Beitrag, dass sie dies unterschiedlich tun, und zwar besonders in Abhängigkeit von ihrer Parteizugehörigkeit und persönlichen Wahrnehmungen. Insofern steht am Ende als zentrale Erkenntnis, dass die in der politischen Kommunikationsforschung als allgegenwärtig angesehene Medienlogik auf Ebene der AkteurInnen keine Konstante ist, sondern von Individuen unterschiedlich stark angenommen wird. Die Forschungsergebnisse lenken somit den Blick auf den voluntaristischen Aspekt dieser Anpassung, der stark durch eine individuelle Effektivitäts-Wahrnehmung mit Blick auf die Möglichkeiten von Parlament und Medien beeinflusst zu sein scheint.

\section{Acknowledgements}

Die Datenbasis dieses Beitrags entstammt dem DFG/ ESF-Projekt "Political Communication Cultures in Western Europe. A Comparative Study" (PF 2016/IO-I). Es wurde innerhalb des ECRP II-Programms der European Science Foundation gefördert und von FWF, FIST, AKA, DFG, MEC, VR, und SNF finanziert. Principal Investigators waren Barbara Pfetsch (Freie Universität Berlin), Juan Díez Medrano (University of Barcelona), Patrick Donges (Universität Greifswald), Anders Esmark (Roskilde University), Nicklas Håkansson (Halmstad University), Otfried Jarren (Universität Zürich), Tom Moring (University of Helsinki), Fritz Plasser (Universität Innsbruck) und Slavko Splichal (University of Ljubljana). Wir danken Prof. Dr. Barbara Pfetsch herzlich für die Möglichkeit zur Sekundäranalyse der Daten.

\section{Literatur}

Altheide, David L. (20I4), Media Edge: Media Logic and Social Reality, New York, NY: Peter Lang.

Altheide, David L. (2004), Media Logic and Political Communication, Political Communication, Vol. 21, 293296.

Altheide, David L./Snow, Robert P. (1979), Media Logic, Beverly Hills, CA: Sage.

Blumler, Jay G./Kavanagh, Dennis (1999), The Third Age of Political Communication: Influences and Features, Political Communication, Vol. 16, 209-230.

Brettschneider, Frank (2002a), Spitzenkandidaten und Wahlerfolg: Personalisierung - Kompetenz - Parteien. Ein internationaler Vergleich, Wiesbaden: VS.
Brettschneider, Frank (2002b), Die Medienwahl 2002: Themenmanagement und Berichterstattung, Aus Politik und Zeitgeschichte, Vol. 49-50, 36-47.

Brüggemann, Michael/Engesser, Sven/Büchel, Florin/ Humprecht, Edda/Castro, Laia (2014), Hallin and Mancini Revisited: Four Empirical Types of Western Media Systems, Journal of Communication, Vol. 64 (6), I037-I065.

Donges, Patrick (2008), Medialisierung politischer Organisationen: Parteien in der Mediengesellschaft, Wiesbaden: VS-Verlag.

Esmark, Anders/Mayerhöffer, Eva (2014), Public AgendaSetting between Media Logic and Political Logic, in: Pfetsch, Barbara (Hg.), Political Communication Cultures in Europe: Attitudes of Political Actors and Journalists in Nine Countries), Houndmills: Palgrave Macmillan, S. 219-245.

Esser, Frank (2013), Mediatization as a Challenge. Media Logic Versus Political Logic, in: Kriesi, Hanspeter/Lavanex, Sandra/Esser, Frank/Matthes, Jörg/Bühlmann, Marc/Bochsler, Daniel (Hg.), Democracy in the Age of Globalization and Mediatization), Houndmills: Palgrave Macmillan, S. 155-I76.

Fawzi, Nayla (2014), Machen Medien Politik? Medialisierung der Energiepolitik aus Sicht von Politikern und Journalisten, Baden-Baden: Nomos.

Früh, Werner (2OII), Inhaltsanalyse: Theorie und Praxis (7., überarb. Aufl), Konstanz: UVK

Hallin, Daniel C./Mancini, Paolo (2004), Comparing Media Systems, Cambridge: Cambridge University Press.

Hayes, Andrew F./Krippendorff, Klaus (2007), Answering the call for a standard reliability measure for coding data, Communication Methods and Measures, I, 77-89.

Heil, Uwe L. (2016), Gesättigte Demokratie: Ein marketingorientierter Alternativbegriff zur Politikverdrossenheit, Wiesbaden: Springer.

Heinisch, Reinhard (2004), Die FPÖ - Ein Phänomen im internationalen Vergleich. Erfolg und Misserfolg des Identitären Rechtspopulismus, Österreichische Zeitschrift für Politikwissenschaft, Vol. 33(3), 247-26I.

Jarren, Otfried/Donges, Patrick (20II), Politische Kommunikation in der Mediengesellschaft, Wiesbaden: VS Verlag für Sozialwissenschaften.

Kepplinger, Hans Mathias (2009), Politikvermittlung, Wiesbaden: VS.

Koch, Thomas/Baden, Christian/Klötzer, Hannah/Müller, Elisabeth (20I3), Bedingt professionell. Eine Untersuchung zur Professionalität der Kommunikationsund Medienarbeit bayerischer Kommunalpolitiker, Studies in Communication / Media, 2(3), 275-295.

Leech, Nancy L./Onwuegbuzie, Anthony L. (2009), A Typology of Mixed Methods Research Designs, Quality \& Quantity, Vol. 43 (2), 265-75. 
Magin, Melanie/Stark, Birgit (2015), Explaining National Differences of Tabloidisation Between Germany and Austria, Journalism Studies, Vol. 16(4), 577-595.

Marcinkowski, Frank/Steiner, Adrian (2009), Was heißt Medialisierung? Autonomiebeschränkung oder Ermöglichung von Politik durch Massenmedien? (Working Paper No. 29), Zürich: National Centre of Competence in Research (NCCR) Challenges to Democracy in the 2Ist Century.

Marcinkowski, Frank (2015), Die „Medialisierung“ der Politik - Veränderte Bedingungen politischer Interessenvermittlung, in: Speth, Rudolf/Zimmer, Annette (Hg.), Lobby Work, Wiesbaden: Springer Fachmedien Wiesbaden, S. 7I-96.

Maurer, Peter/Mayerhöffer, Eva (2009). Themenmanagement von politischen Sprechern und Kommunikationsberatern unter den Bedingungen der Bonner und der Berliner Republik. Ein Zeitvergleich 1994-2008. Zeitschrift für Politikberatung, Vol. 2(3), 447-466.

Maurer, Peter/Vähämaa, Miika (2OI4). Methods and Challenges of Comparative Surveys of Political Communication Elites, in: Pfetsch, Barbara (Hg.), Political Communication Cultures in Europe, Houndmills: Palgrave Macmillan, S. 57-75.

Mazzoleni, Gianpetro/Schulz, Winfried (1999), "Mediatization" of Politics. A Challenge for Democracy? Political Communication, Vol. 16(3), 247-26I.

McLeod, Douglas M./Wise David/Perryman, Mallory. (2017), Thinking About the Media: A Review of Theory and Research on Media Perceptions, Media Effects Perceptions, and Their Consequences, Review of Communication Research 5, 35-83.

Meyer, Thomas (200I), Mediokratie. Die Kolonisierung der Politik durch das Mediensystem, Frankfurt a. M.: edition suhrkamp.

Pfetsch, Barbara (2003), Politische Kommunikationskultur. Politische Sprecher und Journalisten in der Bundesrepublik und den USA im Vergleich, Wiesbaden: Westdeutscher Verlag.

Pfetsch, Barbara/Mayerhöffer, Eva/Moring, Tom (2014), National or Professional? Types of Political Communication Culture Across Europe, in: Pfetsch, Barbara (Hg.), Political Communication Cultures in Europe, Houndmills: Palgrave Macmillan, S. 76-IO2.

Pfetsch, Barbara (2014) (Hg.), Political Communication Cultures in Europe, Houndmills: Palgrave Macmillan.

Pfetsch, Barbara/Donges, Patrick/Plasser, Fritz/Díez Medrano, Juan/Gerstlé, Jacques/Splichal, Slavko/Moring, Tom/ Esmark, Anders/Hakånsson, Nicklas (20I4), Political Communication Cultures in Western Europe, GESIS Datenarchiv, Köln, ZA5ı IO Datenfile Version I.o.o (2OI4), doi:IO.4232/I.5IOI.
Plasser, Fritz/Hüffel, Clemens/Lengauer, Günther (2004), Politische Öffentlichkeitsarbeit in der Mediendemokratie, in: Plasser, Fritz (Hg.), Politische Kommunikation in Österreich, Wiesbaden: VS-Verlag, S. 309-350.

Plasser, Fritz/Ulram, Peter A. (2006), Das Parteiensystem Österreichs, in: Niedermayer, Oskar/Stöss, Richard/ Haas, Melanie (Hg.), Die Parteiensysteme Westeuropas, Wiesbaden: VS-Verlag, S. 35I-372.

Reinemann, Carsten (2010), Medialisierung ohne Ende? Zum Stand der Debatte um Medieneinflüsse auf die Politik. Zeitschrift für Parlamentsfragen, Vol. 57(3), 278293.

Sarcinelli, Ulrich (20II), Politische Kommunikation in Deutschland, Wiesbaden: VS.

Kuhn, Markus (2009), Event-Marketing in der Politik: Medieninszenierungen in Deutschland, Frankreich und der Schweiz, Wiesbaden: VS Verlag für Sozialwissenschaften.

Seiffert, Jens/Fähnrich, Birte (2015), Mehr als nur Rhetorik? Die strategische Kommunikation der deutschen Bundesregierung in der Euro-Krise, in: Fröhlich, Romy/Koch, Thomas (Hg.), Politik - PR - Persuasion), Wiesbaden: Springer, S. 26I-284.

Shehata, Adam/Strömbäck, Jesper (2014), Mediation of Political Realities: Media as Crucial Sources of Information, in: Esser, Frank/Strömbäck, Jesper (Hg.), Mediatization of politics. Understanding the transformation of Western Democracies), London: Palgrave Macmillan, S. 3-28.

Strömbäck, Jesper (2008), Four phases of mediatization: An analysis of mediatization of politics. The International Journal of Press/Politics, Vol. I3(3), 228-246.

Strömbäck, Jesper/Esser, Frank (2014), Mediatization of politics: towards a theoretical framework, in: Esser, Frank/Strömbäck, Jesper (Hg.), Mediatization of politics. Understanding the transformation of Western Democracies), London: Palgrave Macmillan, S. 3-28.

Tenscher, Jens (2012), Nur ZiB und Krone? Medienorientierungen österreichischer Abgeordneter, SWS-Rundschau, Vol. 52(3), 32I-342.

Tenscher, Jens (2003), Professionalisierung der Politikvermittlung? - Politikvermittlungsexperten im Spannungsfeld von Politik und Massenmedien, Wiesbaden: VS.

Walgrave, Stefaan/Van Aelst, Peter (2006), The Contingency of Mass Media's Political Agenda Setting Power. Towards a Preliminary Theory, Journal of Communication, Vol. 56, 88-IO9.

Woelke, Jens (2012), TV-Programmanalyse. Fernsehvollprogramme in Österreich 2OII. Wien: RTR. 


\section{Authors}

Andreas Riedl, Mag. phil., ist wissenschaftlicher Mitarbeiter (prae doc) am Institut für vergleichende Medien- und Kommunikationsforschung (CMC) der Österreichischen Akademie der Wissenschaften (ÖAW) und der Alpen-Adria-Universität Klagenfurt. Sein Dissertationsvorhaben ist an der Schnittstelle von Journalismus- und Medienqualitätsforschung angesiedelt. Weitere Forschungsinteressen umfassen journalistische Selbstverständnisse, Mediatisierungsprozesse und das Wechselspiel zwischen politischen und journalistischen AkteurInnen.

Dr. phil. Peter Maurer arbeitet als Postdoctoral Fellow im Bereich Politische Kommunikation am Institut für Soziologie und Politikwissenschaft der Technisch-Naturwissenschaftlichen Universität Trondheim (NTNU). Von 20II-20I6 war er am Institut für Publizistik der Universität Wien beschäftigt. Seine Schwerpunkte liegen im Bereich politischer Journalismus, politischer Medieneinfluss und politisches Verhalten. Außerdem arbeitet er im Rahmen der COST-Action (IS I308) „Populist Political Communication in Europe" zu populistischen Kommunikationsstrategien.

Birgit Mühl, Mag.a phil., ist Absolventin der Publizistikund Kommunikationswissenschaft an der Universität Wien. In ihrer Magisterarbeit beschäftigte sie sich mit den kognitiven und physiologischen Wirkungen medialer Gewaltdarstellungen. Derzeit arbeitet sie im Projektmanagement für Saferinternet.at bei ISPA - Internet Service Providers Austria, Teil des Safer Internet Centre Austria, dem österreichischen Partner im Safer Internet Netzwerk der EU (Insafe). 